\title{
Emergence of Peregrine solitons in integrable turbulence of deep water gravity waves
}

\author{
Guillaume Michel $^{1},{ }^{*}$ Félicien Bonnefoy ${ }^{2}$, Guillaume Ducrozet $^{2}$, Gaurav Prabhudesai ${ }^{3}$, Annette \\ Cazaubiel $^{4}$, Francois Copie ${ }^{5}$, Alexey Tikan ${ }^{5}$, Pierre Suret ${ }^{5}$, Stéphane Randoux ${ }^{5}$, and Eric Falcon ${ }^{4}$ \\ 1 Institut Jean Le Rond d'Alembert, Sorbonne Université, CNRS, UMR 7190, F-75 005 Paris, France \\ ${ }^{2}$ École Centrale de Nantes, LHEEA, UMR 6598 CNRS, F-44 321 Nantes, France \\ 3 Laboratoire de Physique de l'Ecole normale supérieure, ENS, Université PSL, \\ CNRS, Sorbonne Université, Université Paris-Diderot, Paris, France \\ 4 Université de Paris, Université Paris Diderot, MSC, UMR 7057 CNRS, F-75 013 Paris, France and \\ ${ }^{5}$ Université de Lille, CNRS, UMR 8523 - PhLAM - Physique des Lasers Atomes et Molécules, F-59 000 Lille, France
}

(Dated: August 11, 2020)

\begin{abstract}
We study experimentally the early stages of integrable turbulence of unidirectional deep water gravity waves. By generating partially coherent waves in a $148 \mathrm{~m}$ long wave flume, we observe the emergence of high-amplitude structures formed by nonlinear focusing, commonly referred to as rogue waves. This work confronts the experiment with two recent results obtained in the framework of the nonlinear Schrödinger equation (NLSE), namely that (i) these structures can be locally fitted by a Peregrine soliton and (ii) their emergence leaves a visible trace on the evolution of statistical parameters such as kurtosis. Although Peregrine solitons have been observed for almost ten years in experiments using a deterministic forcing, we report their first systematic study in hydrodynamics with a random forcing. We show that (i) yields accurate results as long as the wave steepness remains moderate, whereas (ii) is very robust and remains valid beyond the assumption of integrability. Numerical simulations of the NLSE and of the fully nonlinear dynamical equations are also performed to support these results.
\end{abstract}

\section{INTRODUCTION}

The statistical properties of the wave fields in oceans are commonly studied in two different frameworks: wave turbulence and integrable turbulence. Introduced in the 1960s, wave turbulence of deep water gravity waves describes isotropic wave fields and shares many similarities with two-dimensional hydrodynamic turbulence [1]. To predict the statistical properties of steady states such as the power-spectral density of the surface elevation, this theory relies on two constant fluxes through scales mediated by four-wave resonant interactions: a flux of energy to small scales and a flux of wave action to large scales $[2,3]$. Half a century after these pioneer theoretical works, its applicability to realistic sea states is being questioned by several experiments [4]. This Rapid Communication investigates the opposite limit of strongly anisotropic wave fields, thereafter assumed to be strictly unidirectional, in which resonant four-wave interactions cannot take place [5]. The dynamics is therefore governed by nonresonant wave interactions and is captured for wave packets of small amplitudes by the focusing nonlinear Schrödinger equation (NLSE). Such random narrow-banded wave packets eventually reach a steady state where neither an energy input nor an energy sink exist, but where statistical features can be described within the framework of the so-called integrable turbulence [6]. As the NLSE describes, to a first approximation, every wave packet of small amplitude and narrow frequency bandwidth undergoing both dispersive and nonlinear effects, integrable turbulence has also been studied in other domains of physics, in particular in optics (see [7-9] and references therein). One of the most striking features of integrable turbulence is the occurrence of high-amplitude events localized in both space and time [9-11], referred to as rogue waves and routinely observed both in oceanography and in nonlinear optics [12].

To reach a steady state that can be described by integrable turbulence, experiments and numerical simulations are initialized with random wave trains whose evolution at short times is either governed (i) by the exponential growth and nonlinear saturation of unstable modes or (ii) by nonlinear self focusing [13]. (i) The first scenario traces back to the modulation instability (MI), introduced in the context of deep water gravity waves by Benjamin and Feir who demonstrated that a continuous wave train is unstable with respect to long-wave perturbation of its envelope [14, 15]. Although the MI has been experimentally studied for a long time in both hydrodynamics and optics [16-18], its transition to integrable turbulence from noise initially superimposed to a monochromatic wave train has only been studied recently, in optics [19, 20] and in hydrodynamics [21]. (ii) In the second scenario, the initial condition is not a monochromatic wave train with some additionnal noise but is made of the linear superposition of various Fourier components. The evolution of such unidirectionnal partially coherent waves has been widely investigated in optical fibers [7-9] and in hydrodynamics [10, 22-26].

* guillaume.michel@sorbonne-universite.fr 


\begin{tabular}{|c|c|c|c|}
\hline Initial steepness $\epsilon(z=0)$ & Final steepness $\epsilon(z=120 \mathrm{~m})$ & Total measurement duration & Local maxima observed \\
\hline 0.057 & 0.056 & $6144 \mathrm{~s}$ & 147 \\
\hline 0.076 & 0.072 & $5120 \mathrm{~s}$ & 184 \\
\hline 0.094 & 0.084 & $5632 \mathrm{~s}$ & 251 \\
\hline
\end{tabular}

TABLE I. Parameters of the various experiments performed: Several samples of $T_{\text {samp }}=512 \mathrm{~s}$ are considered, the initial steepness being imposed at the wave maker whereas the one at $120 \mathrm{~m}$ is measured experimentally. Local maxima are defined in Sect. II.

Despite these numerous works, the mechanisms underlying the dynamics and the statistics of integrable turbulence are still not fully understood. If dispersion is much larger than nonlinearity, by taking into account nonresonant interactions, a modified approach of the standard wave turbulence theory can be used to describe the statistics $[27,28]$. Conversely, some initial conditions are such that dispersion is negligible and the first stages of evolution are dominated by nonlinear self-focusing processes. In that case, humps of the wave envelope undergo nonlinear focusing and would, in the limit of zero dispersion, become singular, which leads to the so-called gradient catastrophes resulting in the emergence of coherent structures of large amplitude. These localized structures in both space and time are prototypes of rogue waves [29].

Previous experiments in wave flumes led to a general consensus on several statistical properties of the transition to integrable turbulence, such as an overshoot of the kurtosis $\kappa[22,23,30,31]$, of the rogue wave probability [24,31] and of the spectral width [25]. The nature of the emergent localized structures has also been investigated: in a basin too short to reach a steady-state, we demonstrated previously that Peregrine solitons (PS) could be observed in such systems but did not provide statistics on that point [32]. Although not explicitly stated, a similar picture can be drawn from the study of single nonlinear pulses [33]. The PS is a well-known analytical solution of the NLSE localized in both space and time domains [34].

This Rapid Communication confronts two recent results obtained in the NLSE to experiments. Result (i), derived by Bertola and Tovbis, predicts that the gradient catastrophe is regularized by structures that can be locally fitted by a PS [35]. Result (ii), obtained numerically, demonstrates that the emergence of these structures connects to the overshoot of the kurtosis [36]. Whereas result (i) has already been verified numerically and qualitatively in experiments $[26,32,36]$, result (ii) has yet to be investigated experimentally.

\section{EXPERIMENTAL RESULTS}

The setup is similar to the one described in Ref. [37]. Experiments are carried out in a wave flume (148 $\mathrm{m}$ long $\times 5 \mathrm{~m}$ wide $\times 3 \mathrm{~m}$ deep) at Ecole Centrale de Nantes. A flap-type wave maker at one end of the basin is driven to generate unidirectional waves of prescribed elevation $\eta_{0}(t)$ in front of it, whereas an absorbing device strongly reduces wave reflection at the opposite end. The surface elevation $\eta(z, t)$ is measured at 20 equally spaced locations $z_{j}=6 \times j \mathrm{~m}(j=1,2, \ldots 20)$, the position of the wave maker being $z=0 \mathrm{~m}$. Resistive wave gauges are used with a sampling frequency of $128 \mathrm{~Hz}$ and a vertical resolution of $0.1 \mathrm{~mm}$. The wave field consists of a one-dimensional (1D) monochromatic carrier wave of frequency $f_{0}=1.15 \mathrm{~Hz}$ (wavelength $\lambda_{0}=1.2 \mathrm{~m}$ ) slowly modulated in phase and in amplitude, the amplitudes being typically of the order of a few centimeters. More precisely, partially-coherent waves are generated with

$$
\eta_{0}(t)=\eta(z=0, t)=\sum_{n=1}^{N} a_{n} \cos \left(2 \pi f_{n} t+\phi_{n}\right), \quad a_{n}=\mathcal{A} \exp \left[-\left(\frac{f_{n}-f_{0}}{2 \Delta f_{0}}\right)^{2}\right],
$$

where $f_{n}=n / T_{\text {samp }}, T_{\text {samp }}=512 \mathrm{~s}$ is the sample length, $N=4096$ is the number of wave components, $\phi_{n}$ is a random phase uniformly distributed in the interval $[0,2 \pi]$ and $\Delta f_{0}=26 \mathrm{mHz}$ is the spectral bandwidth. The constant $\mathcal{A}$ is tuned to fix the steepness $\epsilon(z=0)$ at the wave maker, where

$$
\epsilon(z)=k_{0} \sqrt{2\left\langle\eta(z, t)^{2}\right\rangle}
$$

$k_{0}=2 \pi / \lambda_{0}=\left(2 \pi f_{0}\right)^{2} / g$ is the carrier wave number in deep water condition $\left(k_{0} h=12.3\right), g=9.81 \mathrm{~m} \cdot \mathrm{s}^{-2}$ is the acceleration of gravity and $\langle\cdot\rangle$ denotes an average over time. In this study, we characterize the statistics of three long-time measurement sets for different steepnesses reported in Table I. Each of them consists of several runs in which the wave maker generates the signal of Eq. (1) during $1.2 T_{\mathrm{samp}}$, the front and rear extremities of the recorded wave train then being disregarded. We thereafter discuss both the surface elevation $\eta(z, t)$ and the complex envelope $A(z, t)$ computed with a Hilbert transform and such that

$$
\eta(z, t)=\operatorname{Re}\left[A(z, t) e^{i\left(k_{0} z-\omega_{0} t\right)}\right],
$$



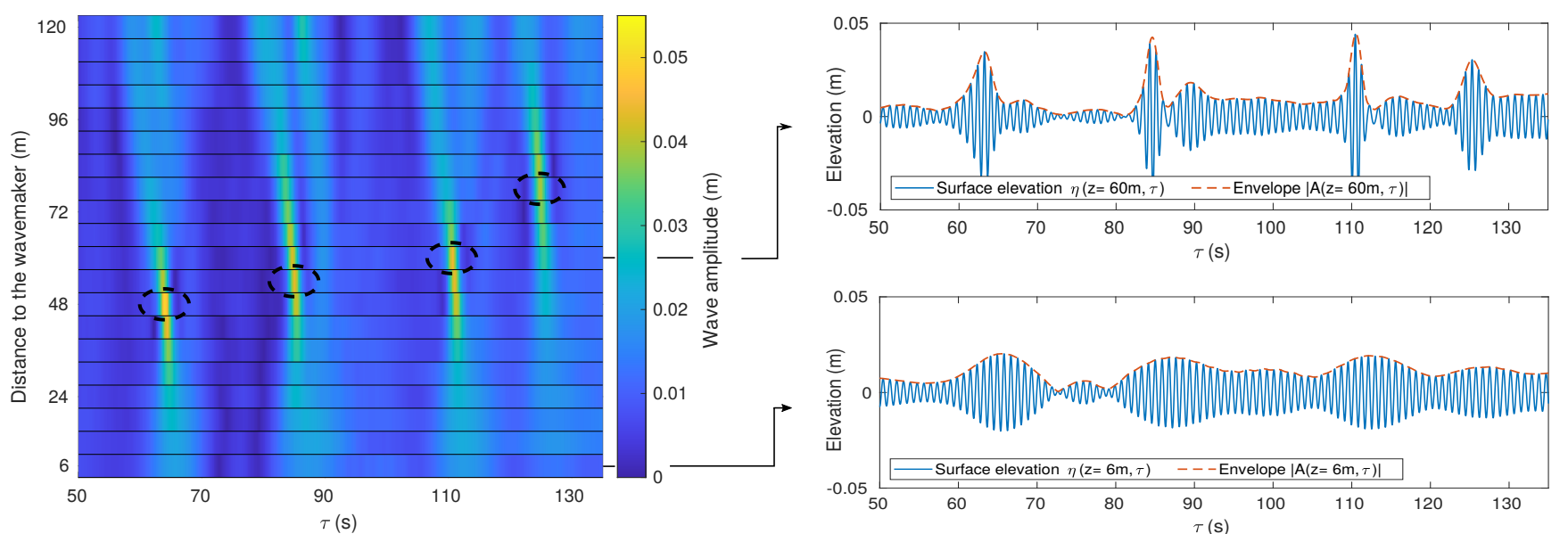

FIG. 1. Spatiotemporal diagram of the wave envelope amplitude $|A(z, \tau)|(\epsilon=0.057)$ inferred from the 20 probes, measurements then being stacked vertically. Local maxima are circled with a dashed line. The time series of the surface elevation $\eta(z, \tau)$ and of the extracted envelope $|A|(z, \tau)$ are also reported for the first and tenth probes $(z=6 \mathrm{~m}$ and $z=60 \mathrm{~m})$.

where $\omega_{0}=2 \pi f_{0}$ is the angular frequency of the carrier and $\operatorname{Re}(X)$ denotes the real part of the complex number $X$. To account for the propagation at the group velocity $c_{\mathrm{g}}=g /\left(4 \pi f_{0}\right)$, we consider the frame of reference moving with $c_{\mathrm{g}}$ and introduce the corresponding time $\tau=t-z / c_{\mathrm{g}}$. Harmonics superimposed on the carrier wave and propagating with the same phase velocity, the so-called bound waves, are filtered out with a bandpass filter of central frequency $f_{0}$ and bandwidth $1 \mathrm{~Hz}$ applied to each elevation signal. Note that this does not affect the spectra in the reported range and has almost no effect on the kurtosis (see below).

Fig. 1 shows the typical space time evolution that is recorded in our experiments with partially coherent waves used as initial condition. It clearly evidences the nonlinear focusing of a few humps of the wave envelope during the first stages of evolution $(0$ to $\sim 60 \mathrm{~m})$, resulting in high amplitude events localized in both space and time domains. For all runs, almost 600 of such events are tracked down systematically by looking for local maxima of $|A(z, \tau)|$ resulting from an increase of at least $50 \%$ of the wave amplitude along the propagation, see four of them identified in Fig. 1 and their overall numbers in Table I. The histogram of their occurrence reported in Fig. 2 confirms that, as expected from a single spatiotemporal diagram, their occurrence peaks at a specific distance from the wave maker around $60 \mathrm{~m}$. Note that wave breaking is observed for the highest steepness from $z \sim 50 \mathrm{~m}$. Whereas the second moment of the surface elevation can be considered as conserved, except for the highest steepness which drops by $10 \%$ along the flume as reported in Table I, Figure 2 also confirms that high amplitude events correlate with an overshoot of the kurtosis defined as

$$
\kappa(z)=\frac{\left\langle\eta(z, t)^{4}\right\rangle}{\left\langle\eta(z, t)^{2}\right\rangle^{2}}
$$
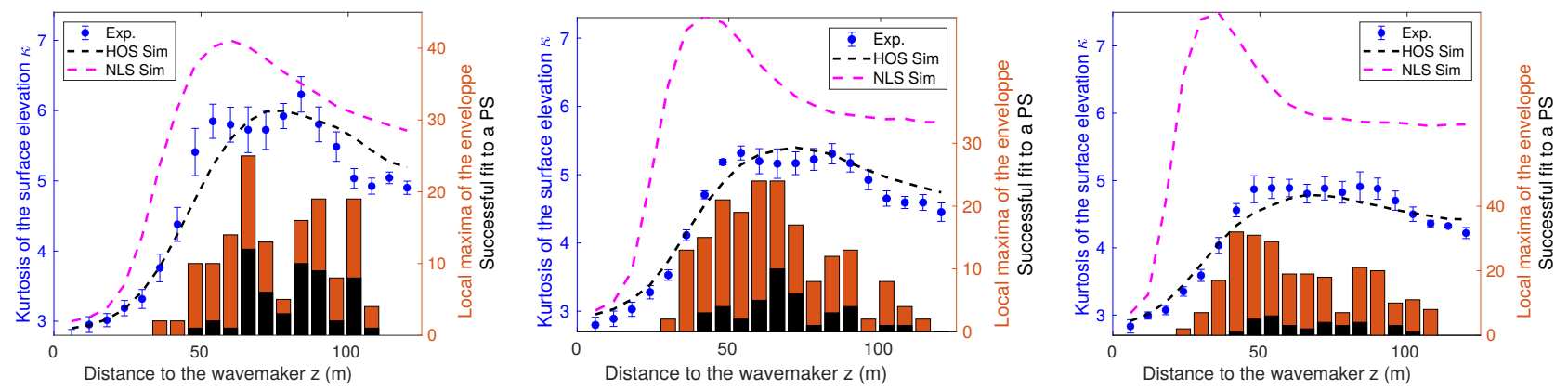

FIG. 2. Statistical properties of the surface elevation along the flume for $\epsilon=0.057$ (left), $\epsilon=0.076$ (center) and $\epsilon=0.094$ (right). The kurtosis measured experimentally is compared to two numerical simulations (NLS and HOS) introduced in Sect. III, and to the experimental histograms of local maxima (all of them in orange bins, only the ones successfully fitted to a Peregrine Soliton in black bins). 


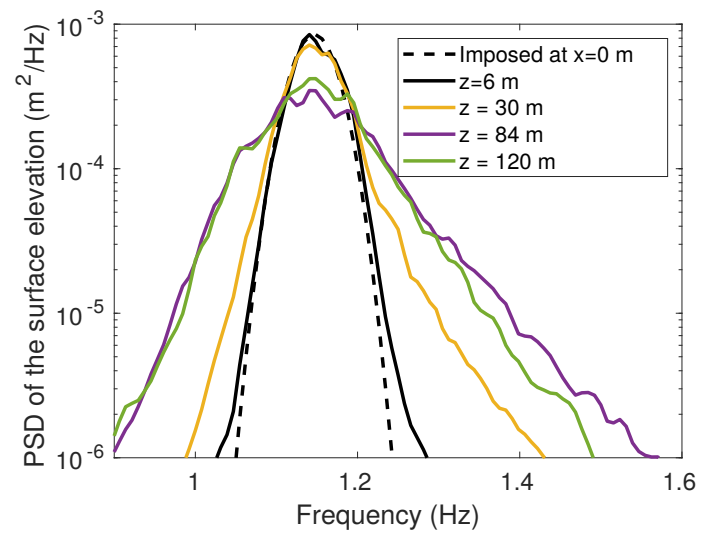

FIG. 3. Power spectral density (PSD) of the surface elevation $\eta(z, t)$ for the smallest steepness $\epsilon=0.057$ at various locations $z$ along the flume. Note the spectral broadening at $z=84 \mathrm{~m}$. Similar overshoots of the spectral bandwidth are observed for the two larger steepnesses.

Finally, the power spectral density (PSD) $S_{\eta}(z, f)$ of the surface elevation is computed at every probe and four of them are reported in Fig. 3 for $\epsilon=0.057$. According to Eq. (1), the wave maker generates a Gaussian PSD of standard deviation $\Delta f_{0}$. As the wave field evolves along the flume, the PSD progressively differs from the initial Gaussian spectrum and a spectral broadening is observed. Moreover, after this strong broadening with distance, the spectral bandwidth then slightly decreases, and therefore overall experiences an overshoot qualitatively similar to the one of the kurtosis.

\section{DISCUSSION}

The experimental results introduced in the previous section make sense in the context of the focusing 1D-NLSE. This equation captures the dynamics of the complex envelope $A(z, \tau)$ in the limit of narrow-banded wave packets of small steepnesses, that is $\Delta f_{0} \ll f_{0}$ and $\epsilon \ll 1$, and reads

$$
i \frac{\partial A}{\partial z}-\frac{k_{0}}{\omega_{0}^{2}} \frac{\partial^{2} A}{\partial \tau^{2}}-\alpha k_{0}^{3}|A|^{2} A=0
$$

The coefficient $\alpha=0.91$ accounts for the finite depth effects that persist even though $k_{0} h=12.3$ [37]. The potential of this model to describe our experiments is introduced by the computation of $\kappa$ from numerical simulations of the NLSE using more samples than experimentally for an improved statistical convergence. As shown in Fig. 2, it qualitatively reproduces the overshoot of $\kappa$ although significant discrepancies resulting from higher-order terms neglected in the NLSE are observed for large steepnesses. The subsequent discussion investigates to what extent other predictions of the NLSE model this experiment, i.e., are robust to a steepness lying out of the limit $\epsilon \rightarrow 0$.

Connection between kurtosis and spectral width. A first consequence of the NLSE is the existence of conserved quantities along the propagation, such as the wave steepness $\epsilon$, in fair agreement with our experiments (see Table I). Exploiting this fact, Onorato et al. derived a relationship between the kurtosis $\kappa_{|A|}$ of the wave envelope and the spectral bandwidth $\Delta f[38]$, that reads in our setup, with $\kappa=(3 / 2) \kappa_{|A|}$ (a result of the narrow bandwidth assumption),

$$
\kappa(z)=\kappa(0)+\frac{3}{\alpha\left(f_{0} \epsilon\right)^{2}}\left(\Delta f(z)^{2}-\Delta f(0)^{2}\right), \quad \kappa(0)=3, \quad \Delta f(0)=\Delta f_{0},
$$

where $\Delta f(z)$ is defined as

$$
\Delta f(z)=\sqrt{\frac{\int S_{\eta}(z, f)\left(f-f_{0}\right)^{2} \mathrm{~d} f}{\int S_{\eta}(z, f) \mathrm{d} f}} .
$$

To test this relationship experimentally, the spectral bandwidth has to be computed over a finite range around the central peak. Unfortunately, in practice, by increasing progressively this range, the measurement noise limit in the PSD is reached before a proper convergence of $\Delta f$ is achieved. Therefore, Eq. (6) can only be qualitatively assessed 

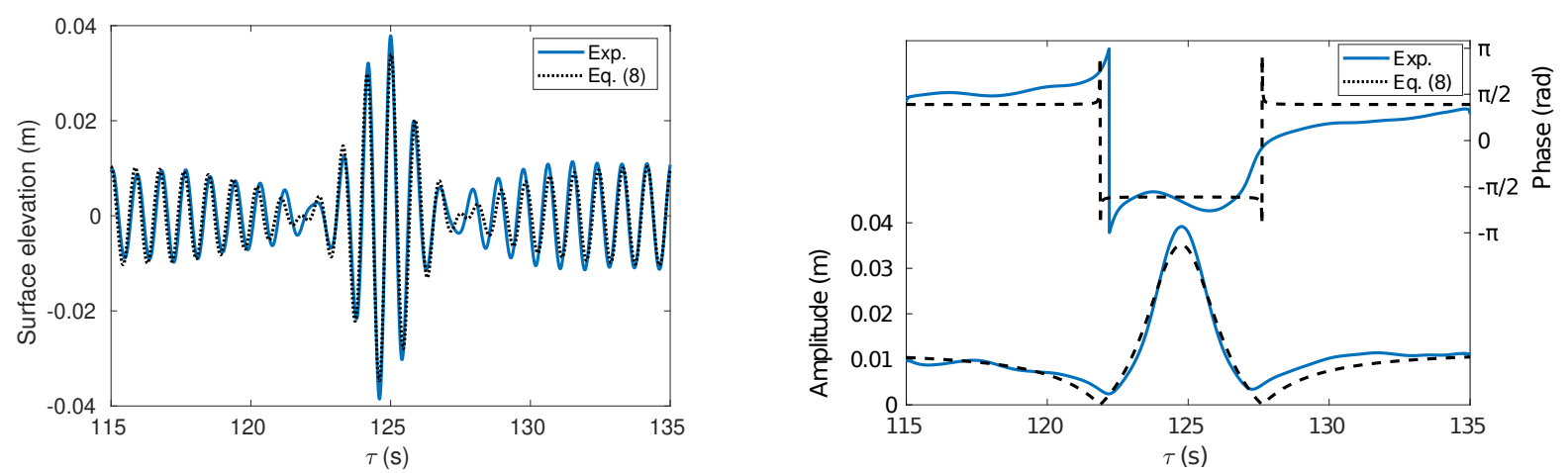

FIG. 4. Fit of the top left circled local maxima of Fig. 1 to a Peregrine soliton (equation (8)). The distance to the wave maker is $z=78 \mathrm{~m}$ and the initial steepness is $\epsilon=0.057$. Both the direct signal $\eta\left(z_{\max }, t\right)$ (left) and the phase and amplitude of the complex envelope $A\left(z_{\max }, t\right)$ (right) are reported, the dashed lines corresponding to the best fit.

by noting that a slight overshoot of the spectral bandwidth is systematically observed (e.g. in Fig. 3), corresponding to the one of the kurtosis.

Coherent structures locally fitted by the Peregrine soliton. Crucially, the NLSE also provides a framework to interpret the evolution of the kurtosis. Indeed, the initial increase of $\kappa$ can be derived for partially coherent wave packets and results from self-focusing [39]: Nonlinearities dominate over linear dispersion and lead to a gradual steepening. Moreover, Bertola and Tovbis showed that the high amplitude structures resulting from the regularization of the gradient catastrophe by dispersion lead to the emergence of Peregrine solitons [35]. This result has also been obtained numerically with the use of large deviation theory [26]. The PS is an analytical solution of the NLSE localized in both space and time, first observed experimentally in the early 2010s in wave flumes [40], in optical fibers [41] and in a plasma [42] with a deterministic forcing. Its generation has been shown to be robust in the presence of noise [43]. We have implemented a procedure where every complex envelope $A\left(z_{\max }, \tau\right)$ of the 582 high amplitude events measured experimentally at various locations $z_{\max }$ is fitted over a 20 seconds window by the PS envelope [34]

$$
f_{\mathrm{PS}}(\tau)=A_{\mathrm{p}}\left[1-\frac{4\left(1-2 i \alpha k_{0}^{3} A_{\mathrm{p}}^{2}\left(z_{\max }-z_{0}\right)\right)}{1+2 \alpha\left[\omega_{0} k_{0} A_{\mathrm{p}}\left(\tau-\tau_{0}\right)\right]^{2}+4\left[\alpha k_{0}^{3} A_{\mathrm{p}}^{2}\left(z_{\max }-z_{0}\right)\right]^{2}}\right] \exp (i \Psi),
$$

where $\tau_{0}$ and $z_{0}$ correspond to the location of its maximal amplitude $3 A_{\mathrm{p}}$ and $\Psi$ is a global phase. The four best-fit parameters $\tau_{0}, z_{0}, A_{\mathrm{p}}$ and $\Psi$ are found numerically. The fit is then considered as being accurate if $\tau_{0}$ and $z_{0}$ match the measured time and position of the maximum amplitude $\left(\left|z_{0}-z_{\max }\right|<3 \mathrm{~m},\left|\tau_{0}-\tau_{\max }\right|<5 \mathrm{~s}\right)$ and if the residuals $\delta \eta(\tau)=\eta\left(z_{\max }, \tau\right)-\operatorname{Re}\left(f_{\mathrm{PS}}(\tau) e^{i\left(k_{0} z_{\max }-\omega_{0} \tau\right)}\right)$ are such that

$$
\frac{\left\langle\delta \eta(\tau)^{2}\right\rangle}{\left\langle\eta\left(z_{\max }, \tau\right)^{2}\right\rangle}<\frac{1}{4} .
$$

An example of such a successful fit for which the left-hand side of Eq. (9) is 0.11 is provided by one of the structures of Fig. 1 and is reported in Fig. 4. It exhibits a $\pi$ phase jump, a characteristic signature of the PS observed experimentally in optics [44] and recently for water waves [32], see also [45]. This approach provides a measure of the degree with which high amplitude structures resulting from nonlinear focusing can be locally fitted by a PS, a key outcome of the present work. The fraction of such accurate fits is reported in Fig. 5 as a function of the steepness, its dependence on the right-hand side of Eq. 9 being indicated by error bars. Although the trend could have been anticipated, it shows that even for the modest steepness $\epsilon=0.057$, half of these events already lie out of the range of validity of the NLSE. This is consistent with the systematic tilt of the structures toward the left reported in Fig. 1, a sign of frequency down shifting captured once $O\left(\epsilon^{4}\right)$ nonlinear terms are considered, e.g. by the so-called Dysthe or modified NLS equation [46]. Similar tilts described by high order corrections have been evidenced on single pulses propagating in a wave flume [33].

Moreover, according to Fig. 2, high-amplitude structures locally similar to PS are found where the kurtosis of the surface elevation $\kappa$ reaches its maximal value. This fact was recently reported from numerical simulations [36], and we thus provide its first experimental demonstration. As the steepness is increased, the PS no longer fits the typical shape of such events, that nonetheless still emerge where the kurtosis peaks. This proves the robustness of the self-focusing mechanism described by the NLSE.

HOS simulations. For high steepnesses, the NLSE no longer provides a quantitative description of our experiments. More specific models including higher nonlinear orders then have to be considered. As a guide, direct numerical 


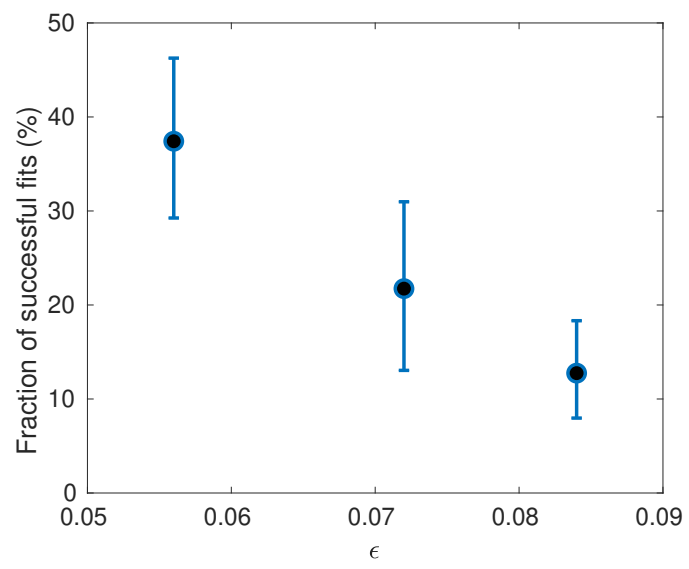

FIG. 5. Every local maxima of the wave envelope is fitted to a Peregrine soliton, see e.g. four of them identified in Fig. 1 and a fit of one of them is shown in Fig. 9. The fraction of successful fits is reported as a function of the initial steepness, evidencing the decrease with $\epsilon$ of the PS as an accurate local model of gradient catastrophe regularization. Negative and positive error bars correspond to the same measure performed with the right-hand side of Eq. (9) being respectively 0.2 and 0.3 instead of $1 / 4$.

simulations of the Euler's equations have been performed with the efficient and accurate High-Order Spectral (HOS) method $[47,48]$. For a direct comparison with the experiments, the numerical model needs to reproduce the main features of the water tank, namely: i) the generation of waves through a wave maker and ii) the absorption of reflected waves with an absorbing beach. To this end a Numerical Wave Tank, entitled HOS-NWT, has been developed [49] (the code being available open-source [50]). It uses the exact same wave maker's motions than in the experiments for a simplified comparison procedure. This specific model has been widely validated in different configurations and more details can be found in $[49,51]$. The results presented in Fig. 2 have been obtained after a careful convergence analysis. The chosen numerical parameters are: HOS order of non-linearity $M=5$ and number of modes free of aliasing errors $N_{x}=3072$. As indicated previously, the experiments exhibit few breaking events. Note that the non-linear potential flow formalism of the HOS-NWT model prevents from direct simulation of overturning waves inducing rotationality and strong viscous effects [52]. The wave breaking is consequently modeled through an adequate procedure in the numerical simulations [53, 54]. Remarkably, the kurtosis computed from our numerical study agrees quantitatively very well with our experiment even for the highest steepness, as shown in Fig. 2.

\section{CONCLUSION}

Experiments in wave flumes provide a reproducible approach to investigate the applicability of simple models to realistic wave fields. The NLSE, in particular, is frequently referred to as a framework capturing high amplitude events localized in both space and time domains. In this study, various predictions of the NLSE have been tested experimentally for partially coherent waves of steepnesses that progressively differ from the range of validity $\epsilon \rightarrow 0$.

On the whole, the general picture of initial self-focusing then regularized by dispersion remains valid, and high amplitude events emerge around a specific distance from the wave maker. For the first time, we report an experimental quantitative measure of the degree with which such events can be locally approximated by Peregrine solitons. Our study shows that this prediction of the NLSE requires extremely small steepnesses. Following a recent numerical study [36], we also carry out the first experimental investigation of the link between the kurtosis of the surface elevation and the emergence of Peregrine-like structures. The correlation between these two quantities is extremely robust and persists for steepnesses as high as 0.1 for which wave breaking occurs.

A long term aim of such experiments is the investigation of the steady states eventually reached. A comparison to the ones of integrable turbulence captured by the NLSE is indeed necessary to ascertain its most robust predictions.

\section{ACKNOWLEDGMENT}

This work was supported by the Agence Nationale de la Recherche (ANR DYSTURB Project No. ANR-17-CE300004). EF thanks partial support from the Simons Foundation/MPS No 651463. FC, AT, PS and SR thank support 
from the Agence Nationale de la Recherche through the LABEX CEMPI project (ANR-11-LABX-0007), the Ministry of Higher Education and Research, Hauts de France council and European Regional Development Fund (ERDF) through the Nord-Pas de Calais Regional Research Council and the European Regional Development Fund (ERDF) through the Contrat de Projets Etat-Region (CPER Photonics for Society P4S).

[1] S. Nazarenko, Wave Turbulence, Lecture Notes in Physics Vol. 82 (Springer, Berlin Heidelberg, 2001).

[2] V. E. Zakharov and N. N. Filonenko, "The energy spectrum for stochastic oscillation of a fluid's surface," Doklady Akad.Nauk 170, 1292-1295 (1967).

[3] V. E. Zakharov and M. M. Zaslavsky, "The kinetic equation and kolmogorov spectra in the weak-turbulence theory of wind waves," Izv. Acad. Sci., USSR, Atmos. Oceanic Phys. (Engl. Transl.) 18, 747 (1982).

[4] E. Falcon, "Laboratory experiments on wave turbulence," Discrete Cont. Dyn. B 13, 819 (2010).

[5] A. I. Dyachenko and V. E. Zakharov, "Is free-surface hydrodynamics an integrable system?" Phys. Lett. A 190, 144 (1994).

[6] V. E. Zakharov, "Turbulence in integrable systems," Stud. Appl. Math. 122, 219 (2009).

[7] P. Walczak, S. Randoux, and P. Suret, "Optical rogue waves in integrable turbulence," Phys. Rev. Lett. 114, 143903 (2015).

[8] P. Suret, R. El Koussaifi, A. Tikan, C. Evain, S. Randoux, S. Christophe, and S. Bielawski, "Single-shot observation of optical rogue waves in integrable turbulence using time microscopy," Nature Communications 7, 13136 (2016).

[9] A. Tikan, S. Bielawski, C. Szwaj, S. Randoux, and P. Suret, "Single-shot measurement of phase and amplitude by using a heterodyne time-lens system and ultrafast digital time-holography," Nature Photonics 12, 228-234 (2018).

[10] M. Onorato, A. R. Osborne, M. Serio, and S. Bertone, "Freak waves in random oceanic sea states," Phys. Rev. Lett. 86, $5831-5834(2001)$.

[11] M. Onorato, A. Osborne, R. Fedele, and M. Serio, "Landau damping and coherent structures in narrow-banded $1+1$ deep water gravity waves," Phys. Rev. E 67, 046305 (2003).

[12] M. Onorato, S. Residori, U. Bortolozzo, A. Montina, and F. Arecchi, "Rogue waves and their generating mechanisms in different physical contexts," Physics Reports 528, 47-89 (2013).

[13] F. Copie, S. Randoux, and P. Suret, "The physics of the one-dimensional nonlinear schrödinger equation in fiber optics: rogue waves, modulation instability and self-focusing phenomena," Reviews in Physics, 100037 (2019).

[14] M. J. Lighthill, "Contributions to the theory of waves in non-linear dispersive systems," J. Inst. Maths Applics 1, 269-306 (1965).

[15] T. B. Benjamin and J. E. Feir, "The disintegration of wave trains on deep water part 1. theory," J. Fluid Mech. 27, 417-430 (1967).

[16] B. M. Lake, H. C. Yuen, H. Rungaldier, and W. E. Ferguson, "Nonlinear deep-water waves: theory and experiment. part 2. evolution of a continuous wave train," J. Fluid Mech. 83, 49-74 (1977).

[17] W. K. Melville, "The instability and breaking of deep-water waves," J. Fluid Mech. 115, 165-185 (1982).

[18] K. Tai, A. Hasegawa, and A. Tomita, "Observation of modulational instability in optical fibers," Phys. Rev. Lett. 56, 135-138 (1986).

[19] M. Närhi, B. Wetzel, C. Billet, S. Toenger, T. Sylverstre, J.-M. Merolla, R. Morandotti, F. Dias, G. Gentry, and J. M. Dudley, "Real-time measurements of spontaneous breathers and rogue wave events in optical fibre modulation instability," Nat. Commun. 7, 13675 (2016).

[20] A. E. Kraych, D. Agafontsev, S. Randoux, and P. Suret, "Statistical properties of the nonlinear stage of modulation instability in fiber optics," Phys. Rev. Lett. 123, 093902 (2019).

[21] A. Chabchoub, G. Genty, J. M. Dudley, B. Kibler, , and T. Waseda, "Experiments on spontaneous modulation instability in hydrodynamics," in Proceedings of The Twenty-Seventh International Ocean and Polar Engineering Conference (San Francisco, ISOPE, Cupertino, 2017) p. 420-424.

[22] M. Onorato, A. Osborne, M. Serio, L. Cavaleri, C. Brandini, and C. T. Stansberg, "Observation of strongly non-gaussian statistics for random sea surface gravity waves in wave flume experiments," Phys. Rev. E 70, 067302 (2004).

[23] M. Onorato, A. Osborne, M. Serio, and L. Cavaleri, "Modulational instability and non-gaussian statistics in experimental random water-wave train," Phys. Fluids 17, 078101 (2005).

[24] M. Onorato, A. R. Osborne, M. Serio, L. Cavaleri, C. Brandini, and C. T. Stansberg, "Extreme waves, modulational instability and second order theory: wave flume experiments on irregular waves," Euro. J. Mech. B Fluids 25, 586-601 (2006).

[25] L. Shemer, A. Sergeeva, and A. Slunyaev, "Applicability of envelope model equations for simulation of narrow-spectrum unidirectional random wave field evolution: Experimental validation," Phys. Fluids 22, 016601 (2010).

[26] G. Dematteis, T. Grafke, M. Onorato, and E. Vanden-Eijnden, "Experimental evidence of hydrodynamic instantons: The universal route to rogue waves," Phys. Rev. X 9, 041057 (2019).

[27] P. A. E. M. Janssen, "Nonlinear four-wave interactions and freak waves," J. Phys. Oceanogr. 33, 863 (2003).

[28] P. Suret, A. Picozzi, and S. Randoux, "Wave turbulence in integrable systems: nonlinear propagation of incoherent optical waves in single-mode fibers," Opt. Express 19, 17852-17863 (2011).

[29] S. Chen, F. Baronio, J. M. Soto-Crespo, P. Grelu, and D. Mihalache, "Versatile rogue wave in scalar, vector, and multidimensional nonlinear systems," J. Phys. A: Math. Theor. 50, 463001 (2017). 
[30] L. Shemer and A. Sergeeva, "An experimental study of spatial evolution of statistical parameters in a unidirectional narrow-banded random wavefield," J. Geophys. Res. 114, C01015 (2009).

[31] L. Shemer, A. Sergeeva, and D. Liberzon, "Effect of the initial spectrum on the spatial evolution of statistics of unidirectional nonlinear random waves," J. Geophys. Res. 115, C12039 (2010).

[32] A. Cazaubiel, G. Michel, S. Lepot, B. Semin, S. Aumaître, M. Berhanu, F. Bonnefoy, and E. Falcon, "Coexistence of solitons and extreme events in deep water surface waves," Phys. Rev. Fluids 3, 114802 (2018).

[33] A. Chabchoub, N. Hoffmann, M. Onorato, G. Genty, J. M. Dudley, and N. Akhmediev, "Hydrodynamic Supercontinuum," Phys. Rev. Lett. 111, 054104 (2013).

[34] D. H. Peregrine, "Water waves, nonlinear schrödinger equations and their solutions," J. Aust. Math. Soc. Ser. B, Appl. Math. 25, 16 (1983).

[35] M. Bertola and A. Tovbis, "Universality for the focusing nonlinear schrödinger equation at the gradient catastrophe point: Rational breathers and poles of the tritronquée solution to painlevé i," Comm. Pure Appl. Math. 66, 678-752 (2013).

[36] A. Tikan, "Effect of local peregrine soliton emergence on statistics of random waves in the 1-d focusing nonlinear schrödinger equation," Phys. Rev. E 101, 012209 (2020).

[37] F. Bonnefoy, A. Tikan, F. Copie, P. Suret, G. Ducrozet, G. Prabhudesai, G. Michel, A. Cazaubiel, E. Falcon, G. El, and S. Randoux, "From modulational instability to focusing dam breaks in water waves," Phys. Rev. Fluids 5, 034802 (2020).

[38] M. Onorato, D. Proment, G. El, S. Randoux, and P. Suret, "On the origin of heavy-tail statistics in equations of the nonlinear schrödinger type," Phys. Lett. A 380, 3173-3177 (2016).

[39] G. Roberti, G. El, S. Randoux, and P. Suret, "Early stage of integrable turbulence in the one-dimensional nonlinear schrödinger equation: A semiclassical approach to statistics," Phys. Rev. E 100, 032212 (2019).

[40] A. Chabchoub, N. P. Hoffmann, and N. Akhmediev, "Rogue wave observation in a water wave tank," Phys. Rev. Lett. 106, 204502 (2011).

[41] B. Kibler, J. Fatome, C. Finot, G. Millot, F. Dias, G. Genty, N. Akhmediev, and J. M. Dudley, "The peregrine soliton in nonlinear fibre optics," Nature Phys. 6, 790-795 (2010).

[42] H. Bailung, S. K. Sharma, and Y. Nakamura, "Observation of peregrine solitons in a multicomponent plasma with negative ions," Phys. Rev. Lett. 107, 255005 (2011).

[43] A. Chabchoub, "Tracking Breather Dynamics in Irregular Sea State Conditions," Phys. Rev. Lett. 117, 144103 (2016).

[44] A. Tikan, C. Billet, G. El, A. Tovbis, M. Bertola, T. Sylvestre, F. Gustave, S. Randoux, G. Genty, P. Suret, and J. M. Dudley, "Universality of the peregrine soliton in the focusing dynamics of the cubic nonlinear schrödinger equation," Phys. Rev. Lett. 119, 033901 (2017).

[45] G. Xu, K. Hammani, A. Chabchoub, J. M. Dudley, B. Kibler, and C. Finot, "Phase evolution of peregrine-like breathers in optics and hydrodynamics," Phys. Rev. E 99, 012207 (2019).

[46] J. D. Carter, D. Henderson, and I. Butterfly, "A comparison of frequency downshift modelsof wave trains on deep water," Phys. Fluids 31, 013103 (2019) .

[47] D. G. Dommermuth and D. K. Yue, "A high-order spectral method for the study of nonlinear gravity waves," J. Fluid Mech. 184, 267-288 (1987).

[48] B. J. West, K. A. Brueckner, R. S. Janda, D. M. Milder, and R. L. Milton, "A new numerical method for surface hydrodynamics," J. Geophys. Res. 92, 11803-11824 (1987).

[49] G. Ducrozet, F. Bonnefoy, D. Le Touzé, and P. Ferrant, "A modified high-order spectral method for wavemaker modeling in a numerical wave tank," Eur. J. Mech. B. Fluids 34, 19-34 (2012).

[50] Ecole Centrale Nantes, LHEEA, Open-source release of HOS-NWT, https://github.com/LHEEA/HOS-NWT.

[51] F. Bonnefoy, G. Ducrozet, D. Le Touzé, and P. Ferrant, "Advances in numerical simulation of nonlinear water waves," (World Scientific, 2010) Chap. Time domain simulation of nonlinear water waves using spectral methods, p. 129-164.

[52] G. Ducrozet, F. Bonnefoy, and Y. Perignon, "Applicability and limitations of highly non-linear potential flow solvers in the context of water waves," Ocean Eng. 142, 233-244 (2017).

[53] B. R. Seiffert, G. Ducrozet, and F. Bonnefoy, "Simulation of breaking waves using the high-order spectral method with laboratory experiments: Wave-breaking onset," Ocean Model. 119, 94-104 (2017).

[54] B. R. Seiffert and G. Ducrozet, "Simulation of breaking waves using the high-order spectral method with laboratory experiments: wave-breaking energy dissipation," Ocean Dynam. 68, 65-89 (2018). 